\title{
Instability and excessive mechanical loading mediate subchondral bone changes to induce osteoarthritis
}

\author{
Jianxi Zhu ${ }^{1,2}$, Yong $\mathrm{Zhu}^{1,2}$, Wenfeng Xiao ${ }^{1,2}$, Yihe $\mathrm{Hu}^{1,2}$, Yusheng $\mathrm{Li}^{1,2}$ \\ ${ }^{1}$ Department of Orthopaedics, ${ }^{2}$ National Clinical Research Center for Geriatric Disorders, Xiangya Hospital, Central South University, Changsha \\ 410008, China \\ Contributions: (I) Conception and design: J Zhu; (II) Administrative support: Y Li, Y He; (III) Provision of study materials or patients: Y Zhu; (IV) \\ Collection and assembly of data: W Xiao; (V) Data analysis and interpretation: J Zhu; (VI) Manuscript writing: All authors; (VII) Final approval of \\ manuscript: All authors. \\ Correspondence to: Yusheng Li; Yihe Hu. Xiangya Hospital, Central South University, No. 87, Xiangya Rd., Changsha 410008, China. \\ Email: liyusheng@csu.edu.cn; huyh1964@163.com.
}

Background: To assess the diversified effects of mechanical instability, excessive mechanical loading on subchondral bone remodeling. And to investigate the underlying cartilage degeneration and osteoarthritis (OA) progression in ipsilateral and contralateral knees, given that OA progression always affects joints bilaterally.

Methods: Anterior cruciate ligament transection (ACLT) of the left knee was used to induce OA in C57/ B6 mice for 1, 3 and 6 months. Both left (ipsilateral) and right (contralateral) knees underwent microcomputerized tomography (micro-CT) scan and morphological analysis. The subchondral bone metabolism analysis by immunostaining of tartrate-resistant acid phosphatase (TRAP) and Osterix. Behavioral analyses including von Frey test and CatWalk gait analysis were also performed. Western blot analysis was performed to assess the signaling pathways involved in OA progression.

Results: Analyses showed that various changes in ipsilateral and contralateral knees lead to OA progression. Articular cartilage was rapidly destroyed on the ipsilateral side but was only gradually destroyed on the contralateral side. Micro-CT data showed a rapid decrease with a subsequent partial recovery of bone volume in the late stage on the ipsilateral side, while a gradual condensation of bone density was seen on the contralateral side. Immunostaining showed increased osteoclastic and osteoblastic activity in the early stage on the ipsilateral side, but only slight osteoblastic changes on the contralateral side. Behavioral analyses including von Frey and gait analysis showed that contralateral knees compensate ipsilateral mechanical loading, but also that this mechanism failed to work in the late stage.

Conclusions: Diversified mechanical loading properties lead to OA progression through different mechanisms of subchondral bone remodeling. Acute ACLT led to OA through bone density reduction, while the contralateral side developed OA gradually due to subchondral bone sclerosis.

Keywords: Osteoarthritis (OA); mechanical loading; subchondral bone; contralateral side

Submitted Nov 28, 2019. Accepted for publication Jan 18, 2020.

doi: $10.21037 /$ atm.2020.02.103

View this article at: http://dx.doi.org/10.21037/atm.2020.02.103

\section{Introduction}

Osteoarthritis (OA) is the most prevalent degenerative joint disease and leading cause of physical disability. It is predicted to affect 67 million people in the United States by the year 2030 (1-3) with an economic cost of $1-2.5 \%$ of gross national product in USA. Seniors are at high risk for OA, and the prevalence of OA increases significantly with age. Clinical changes observed in OA mainly include wear on articular cartilage, sclerosis of subchondral bone, osteophyte formation, joint space narrowing, joint deformity and synovial hypertrophy among others. Various 
etiologies lead to a final clinical presentation of OA. Although risk factors like obesity, joint trauma, mechanical loading, instability of surrounding tissue, osteoporosis and genetic predispositions and heritable factors have been identified, the exact pathogenesis of OA remains largely unknown (4). Because of this uncertainty, there are no commonly accepted disease-modifying anti-rheumatic drugs used for OA (5), and the only effective treatment for advanced stage $\mathrm{OA}$ is arthroplasty (6).

Articular cartilage destruction is the most prominent characteristic and major clinical presentation of OA. A number of factors have been identified to be responsible for pathologic alterations in osteoarthritic cartilage. Hypoxiainducible factor- $2 \alpha$ (HIF-2 $\alpha)$ (7), complement component 5 (C5) (8) and matrix metalloproteinase 13 (MMP13) have been found to affect articular cartilage destruction. The homeostasis and integrity of articular cartilage are dependent on its biochemical and biomechanical interplay with subchondral bone and other joint tissues (9). Subchondral bone has become a focus of recent research because of its significant modification in OA progression. This thin layer of bone underneath the cartilage provides mechanical support for articular cartilage during the mechanical loading process (10). Clinically, disruption of the tidemark and subchondral bone sclerosis can be seen with articular cartilage destruction at the osteochondral junction (11).

There is increasing evidence that subchondral bone remodeling is coupled with articular cartilage deterioration (12). Despite the anatomical link between subchondral bone and articular cartilage, their roles and interactions in OA progression remain poorly defined (13). Studies provide new evidence that physiological levels of mechanical loading are necessary for normal cartilage renewal (14) and subchondral bone remodeling (15). The presence of bone marrow lesions is highly associated with OA progression and especially with the development of osteoarthritic pain (16). The continuous microstructural link between articular cartilage and subchondral bone allows cytokines to diffuse between these. Transforming growth factor (TGF)- $\beta$ is one of the most important factors expressed in subchondral bone and articular cartilage. Its upregulation is involved in the destruction of both structures (12).

Mechanical loading plays a central role in skeletal homeostasis and pathogenesis (17). Physiological levels of mechanical loading are essential for cartilage metabolism and homeostasis. In vivo studies have found long-term tissue changes associated with growth, remodeling, and aging by simulating physiological loading conditions, demonstrating that a normal range of mechanical loading is beneficial to cartilage renewal (4). Ex vivo cyclic compression at frequencies of 0.01 to $1.00 \mathrm{~Hz}$ can stimulate proteoglycanlike aggrecan biosynthesis, but stimulation with lower frequencies and amplitudes seems to have no significant effect on cartilage proteoglycan biosynthesis (18). A cascade of proinflammatory cytokines including interleukin-1 (IL-1), interleukin-6 (IL-6) and tumor necrosis factor (TNF)- $\alpha$ can be overexpressed in osteoarthritic joints upon aberrant mechanical loading. These proinflammatory factors are strong driving forces of cartilage matrix degeneration and periarticular inflammation (19). These cytokines disturb the normal remodeling activities of chondrocytes by increasing the production of matrix metalloproteinases and aggrecanases which respectively cleave collagen and proteoglycan and decrease the synthesis of extracellular matrix components. Chondrocytes can produce autocrine and paracrine proinflammatory cytokines in response to excessive mechanical loading or instability. These inflammatory mediators are important factors in the progression of OA and OA pain.

Unraveling this complex interaction between articular cartilage and underlying subchondral bone is complicated by the fact that mechanical loading is both a major risk factor for OA progression and a cause of local subchondral bone mechanical adaptation. Mechanical loading can positively or negatively influence subchondral bone metabolism, which in turn exerts effects on articular cartilage. The temporospatial sequence of these events, however, remains questionable. Here, we provide our point of view on the crosstalk between articular cartilage and subchondral bone in knee joints subjected to mechanical instability and overloading. Based on our findings, we propose that the pathogenesis of OA could be a synergistic effect of mechanical instability and mechanical overloading in both the directly involved ipsilateral knee and the contralateral knee.

\section{Methods}

\section{Animal model}

Anterior cruciate ligament transection (ACLT) was performed on 12-week old male C57/B6 mice. All the mice were housed at $21 \pm 2{ }^{\circ} \mathrm{C}$ with 12 -hour light/dark cycles ( $n=8$ /group). All procedures complied with the Animals (Scientific Procedures) Act 1986 and received university ethical approval of Xiangya Hospital (2019030186) (20). For ACLT surgery, a $10 \mathrm{~mm}$ incision on the patella was made to 
expose patella and patellar tendon. The joint capsule medial to the patellar tendon was incised with a \#15 blade. Blunt dissection and a flip flap of the patella were then performed to expose the intercondylar region, providing an acceptable surgical view of the anterior cruciate ligament region. Hemorrhage from the surrounding soft tissue due to blunt dissection was controlled using pressure with absorbent materials. The anterior cruciate ligament was transected with a micro-surgical knife under direct visualization, avoiding the posterior cruciate ligament. Complete transection was validated by a positive anterior drawer test. The joint capsule was closed with a separate 6-0 suture and the subcutaneous layer with a 7-0 cutting suture. The skin was closed by a 6-0 suture. Immediately after surgery, the mice were kept warm with heat pads. Two days after surgery, all the mice regained acceptable physical mobility.

\section{Histology}

Mice were euthanized with carbon dioxide, with the procedure done over $20 \mathrm{~min}$. After perfusion with heparinized phosphate-buffered saline (PBS) and paraformaldehyde, the knee joints were fixed in $4 \%$ paraformaldehyde for $24 \mathrm{~h}$. After micro-CT scanning, the whole joints were decalcified in $10 \%$ ethylenediaminetetraacetic acid (EDTA) with a $\mathrm{pH}$ of 7.4 for 14 days in a shaker. Joints were embedded in paraffin and $4 \mathrm{~mm}$ sagittal sections were made through the entire medial joint at $40 \mathrm{~mm}$ intervals. The paraffin sections were stained with Safranin-O and fast green and used for immunohistology. Immunostaining was performed using a standard protocol. The Osteoarthritis Research Society International (OARSI) score was assessed using a previously described standard protocol (21). We incubated sections with primary antibodies to mouse Osterix (Abcam, 1:200, ab22552) and tartrate-resistant acid phosphatase (TRAP) (Abcam, 1:200, ab133238) overnight at $4^{\circ} \mathrm{C}$. For immunohistochemical staining, a horse radish peroxidasestreptavidin detection system (Dako) was used to detect immunoactivity, followed by counterstaining with hematoxylin (Sigma-Aldrich).

\section{von Frey test}

Electronic von Frey test for hypersensitivity to pain was assessed before operation and at 2, 4, 8, 12, 16, 20 and 24 weeks following ACLT. Animals were placed in acrylic cages $(12 \mathrm{~cm} \times 10 \mathrm{~cm} \times 17 \mathrm{~cm}$ high) in a quiet room $30 \mathrm{~min}$ before tests. The electronic pressure meter consisted of a force transducer fitted with polypropylene tips with various forces (electronic von Frey anesthesiometer, IITC Inc., Life Science Instruments, Woodland Hills, CA, USA). Two researchers carried out the testing. One researcher used the polypropylene tip to stimulate perpendicularly one of the five distal footpads of the hind paw. Stimulations were performed when animals were quiet, not urinating nor defecating and not resting on their paws. The second researcher observed the maximal intensity of the stimulus automatically recorded when the paw was withdrawn. The test was repeated three times for each mouse and the mean value was used. Results were interpreted as the average value of three withdrawal threshold force (g) measurements.

\section{CatWalk gait analysis}

Gait analysis was conducted at 1, 3 and 6 months after ACLT using the CatWalk (Noldus Information Technology, Wageningen, Netherlands) platform. Mice were placed on a translucent glass plate walkway and allowed to walk freely without restriction. A completely internally reflected green fluorescent light turned on when force was applied to the glass. Paw prints were captured by a high speed video camera under the walkway and were analyzed by computer software (CatWalk XT 9). At least three complete and uninterrupted walks were recorded for each mouse. Mouse selection was done by two independent observers (J.Z. and W.S.) who were blinded to surgical intervention status. Stance time and maximal intensity were collected for statistical analysis.

\section{Micro-CT}

After fixation, we analyzed the knee joints by high resolution micro-CT (SkyScan). The scanner was set to a voltage of $65 \mathrm{kVp}$, a current of $135 \mu \mathrm{A}$ and a resolution of $5.25 \mu \mathrm{m}$ per pixel. Sagittal views of the tibial subchondral bone were used to perform three-dimensional reconstruction and analyses. We reconstructed and analyzed images using NRecon v1.6 and CTAn v1.9. Three-dimensional model visualization software, CT Vol v2.0, was used to analyze parameters of the trabecular bone in the tibial subchondral bone area. Five consecutive representative images were chosen to reconstruct three-dimensional views. Threedimensional structural parameters analyzed included trabecular bone volume per tissue volume (BV/TV) and trabecular pattern factor (Tb.Pf). 

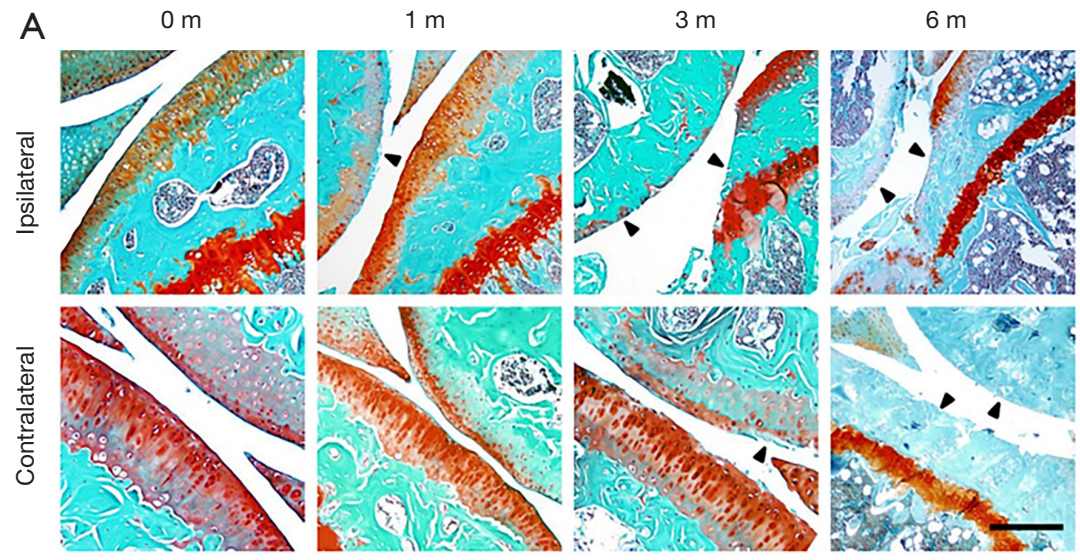

B

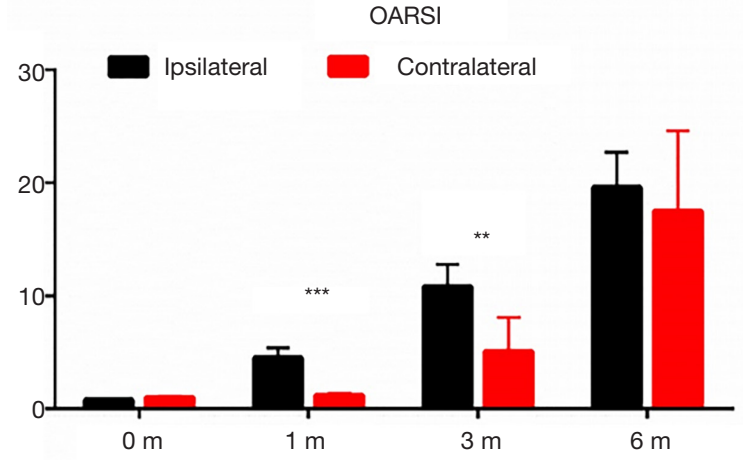

Figure 1 Ipsilateral and contralateral knees developed OA in distinct patterns in murine ACLT model. (A) Representative photos of Safranin $\mathrm{O}$ and fast green staining of ipsilateral (upper) an contralateral side of knee joints 0, 1, 3 and 6 months after ACLT; (B) statistical analysis, $\mathrm{n}=8$ per group, statistical significance was determined by unpaired Student's $t$ test and all data were shown as a bar with means \pm standard deviations. Scale bars, $500 \mu \mathrm{m}$. **, $\mathrm{P}<0.01$, ***, $\mathrm{P}<0.001$ compared with the sham operated group at different time points. ACLT, anterior cruciate ligament transection; OA, osteoarthritis.

\section{Western blot}

Western blotting was performed on the tibial subchondral bone marrow. The samples were separated by SDS-PAGE gel and transferred onto a nitrocellulose membrane. After incubation with specific primary and secondary antibodies, signal was detected by an enhanced chemiluminescence kit (Amersham Biosciences). The primary antibodies used were as follows: rabbit anti-Osterix (Abcam), rabbit anti-pSMAD 2/3 (Abcam) and rabbit anti-GAPDH $(1: 1,000,5174$, Cell Signaling Technology). The experiments were repeated three times and a representative film was selected.

\section{Statistical analysis}

Data were calculated as means $\pm 95 \%$ CIs. A normality distribution test was performed with GraphPad Prism
8.0 on all the datasets, for which $\mathrm{P}$ values $<0.05$ were considered significant. We used Shaprio-Wilk test before ANOVA to ensure the gaussian distribution of all samples. The statistical significance of histological, von Frey results micro-CT data was analyzed using one-way analysis of variance (ANOVA) with Bonferroni's test. A P value $<0.05$ was considered statistically significant.

\section{Results}

Ipsilateral and contralateral knees developed $\mathrm{OA}$ in distinct patterns in a murine ACLT model

We first examined the temporal change pattern of articular cartilage in both ipsilateral and contralateral knees following ACLT. The Safranin-O and fast green staining showed proteoglycan loss and articular cartilage degeneration in both 
ipsilateral and contralateral knees. The starting point and progression pattern, however, differed between ipsilateral and contralateral joints (Figure 1A). The OARSI score revealed that time-dependent articular cartilage deterioration was evident 1 month after ACLT. Cartilage wear and proteoglycan loss were significant after 3 months at both the tibial plateau and femoral condyle. Six months post-ACLT, the posterior part of the tibial cartilage had nearly completely degenerated due to the anterior shift of the tibial plateau caused by the procedure. The anterior part of the tibial cartilage, however, remained relatively preserved.

The contralateral knee developed OA in a different temporospatial pattern. At 1 month after ACLT, no significant proteoglycan loss or articular cartilage degeneration was observed. Cartilage wear was seen at 3 months post-ACLT. Compared to the ipsilateral side, the entire tibial plateau and femoral condyle had degenerated after 6 months (Figure 1B).

Morphological changes in subchondral bone also presented in two different patterns for ipsilateral and contralateral sides. Trabecular bone and the subchondral bone plate on the contralateral knee acutely deteriorated 1 month after ACLT. After 6 months, the posterior tibial plateaus had collapsed and the bone in the area was condensed. The trabecular architecture in ipsilateral knees, in contrast, had gradually condensed over time. Although the trabecular bone was not obviously affected 1 month after ACLT, it was significantly condensed after 3 months. After 6 months post-ACLT, contralateral subchondral bones were nearly completely condensed, with evident sclerosis in both the femoral condyle and tibial plateau; the trabecular architecture could barely be seen (Figure $1 A$ ).

The animals' behavior correlated with the progression of OA. Hypersensitivity at their paws indicated the level of OA pain in the progression of the disease. We found the initial threshold for both paws of naïve mice were approximately 4 g. Immediately after ACLT, the ipsilateral paw reached peak hypersensitivity at around $1 \mathrm{~g}$, then gradually desensitized with time. The hypersensitivity of the contralateral paw was completely different. It barely changed in the early period after ACLT (around 2 to 4 weeks), then gradually sensitized after 8 weeks. The paw withdrawal threshold of the contralateral paw was still higher than that of the ipsilateral side at 24 weeks, but the difference between the two was significantly reduced. The distinct pattern of OARSI and electronic von Frey tests indicated acute progression of $\mathrm{OA}$ in the ipsilateral knee and a gradual progression in the contralateral knee.

\section{Behavioral analysis reveals mechanical loading changes in $O A$ progression of both knees}

To identify the potential mechanism leading to the contralateral changes in cartilage and subchondral bone architecture, we undertook CatWalk gait analyses to assess ipsilateral and contralateral gait changes due to altered mechanical loading (22). There was no significant difference in stance or maximal contact maximal intensity in each treatment group before ACLT. One month after ACLT, the ipsilateral knees showed significantly lower stance time and maximal intensity compared to contralateral knees and controls. At this timepoint, the contralateral knees showed significantly higher levels of stance time and maximal contact maximal intensity as a means of compensation. At 3 and 6 months after ACLT, values measured in ipsilateral and contralateral knees approached the same level, but remained significantly different from those taken from the sham operation group (Figure 2). These results indicate that ipsilateral knees show a significant reduction in mechanical loading in the early stage of OA progression and gradually recover in the later stage of OA. Contralateral knees compensated for the early decrease in mechanical loading of the ipsilateral knee.

\section{Differential subchondral bone architecture in ipsilateral and contralateral knees after ACLT}

Based on previous findings that abnormal mechanical loading can change subchondral structures, we further analyzed bony microarchitectural changes using microCT (23). We first compared subchondral bone BV/TV and Tb.Pf before ACLT and found no differences between groups (Figure 3). This result confirmed left and right joint symmetry in naïve mice at baseline. Analyses of ipsilateral knees 1 month after ACLT showed significant degeneration as evidenced by decreased BV/TV and increased Tb.Pf. No obvious subchondral bone change was seen at this point (Figure 3). At 3 and 6 months after ACLT, the ipsilateral knees tended to exhibit an unbalanced distribution of bone density, showing decreased bone density anteriorly and 


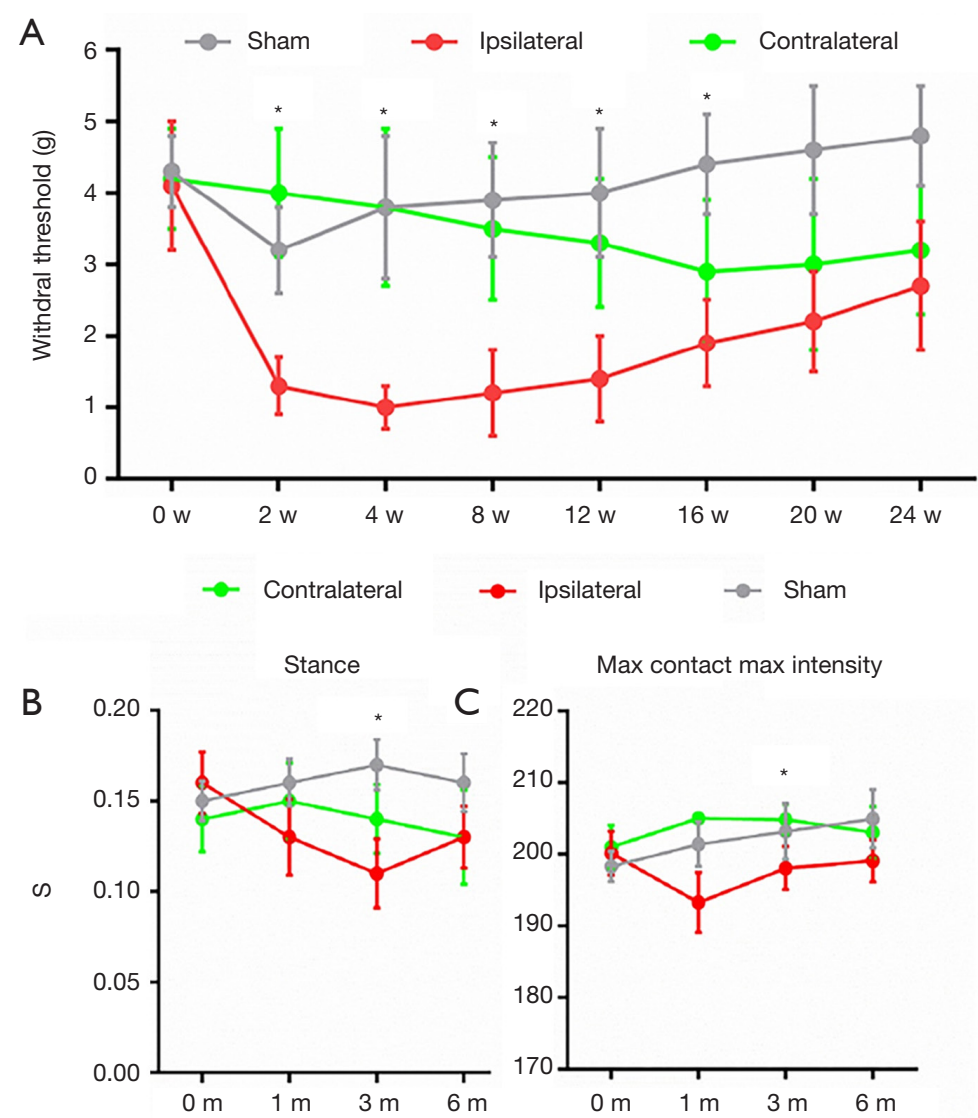

Figure 2 Behavioral analysis reveals mechanical loading changes in OA progression of both knees. (A) von Frey analysis showing hind paw withdrawal threshold of sham operated mice, ipsilateral and contralateral side of mice in different time points after ACLT; CatWalk gait analysis including stance (B) and max contact max intensity $(\mathrm{C})$ of sham operated mice, ipsilateral and contralateral side of mice in different time points after ACLT. $\mathrm{n}=8$ per group, statistical significance was determined by multifactorial ANOVA and all data were shown as plots with means \pm standard deviations. ${ }^{*}, \mathrm{P}<0.05$ compared with the sham operated group at different time points. ACLT, anterior cruciate ligament transection; OA, osteoarthritis.

increased bone density posteriorly (Figure $3 A$ ).

The decreased BV/TV and increased Tb.Pf were recovered to different degrees 1 month after ACLT. Contralateral knee bone density was significantly increased at both the femoral condyle and tibial plateau as evidenced by higher BT/TV compared to the sham group. The Tb.Pf of the contralateral knee was increased due to the loss of bone contiguity and microarchitecture (Figure 3B). These data indicate that subchondral bone degeneration in ipsilateral and contralateral knees progressed in different patterns. Acute abnormal mechanical loading in the ipsilateral knee resulted in decreased bone density and contralateral compensation resulted in a gradual sclerosis in subchondral bone.

\section{Differential osteoblastic and osteoclastic activities in ipsilateral and contralateral knees after ACLT}

We next examined subchondral bone remodeling patterns in ipsilateral and contralateral knees in response to different mechanical loading properties. We evaluated osteoclastic and osteoblastic activity of subchondral bone at different timepoints after ACLT by immunostaining of TRAP and Osterix. For the sham operation group, both osteoclastic and osteoblastic activity remained relatively low (Figure 4). In ipsilateral knees, osteoclastic activity increased rapidly after ACLT and peaked 1 month post surgery. Osteoblastic activity was also elevated 1 month after ACLT. In contralateral knees, 
A
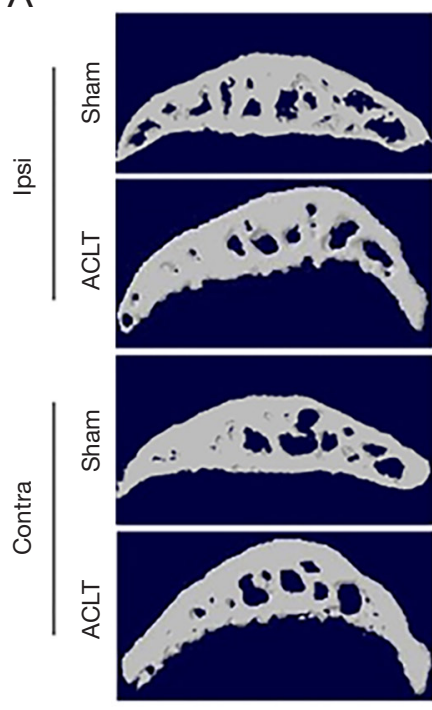

Ipsilateral sham

B

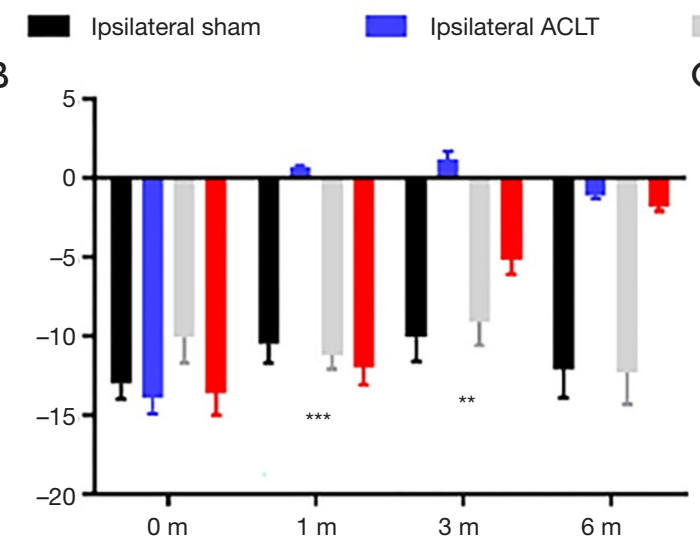

$1 \mathrm{~m}$
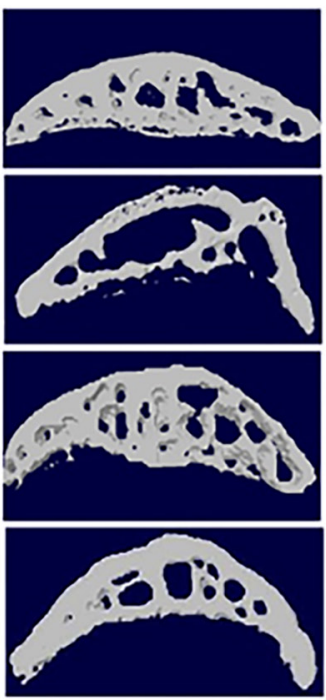

$3 m$
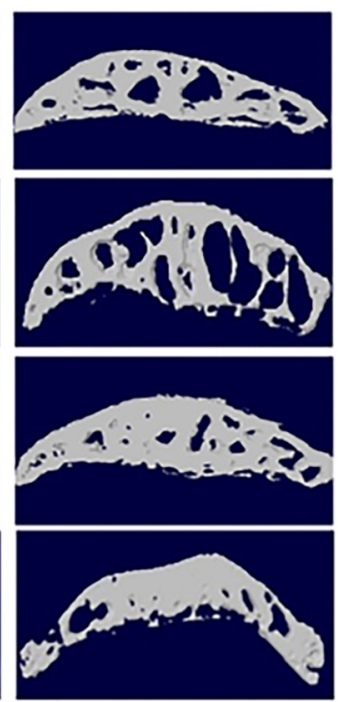
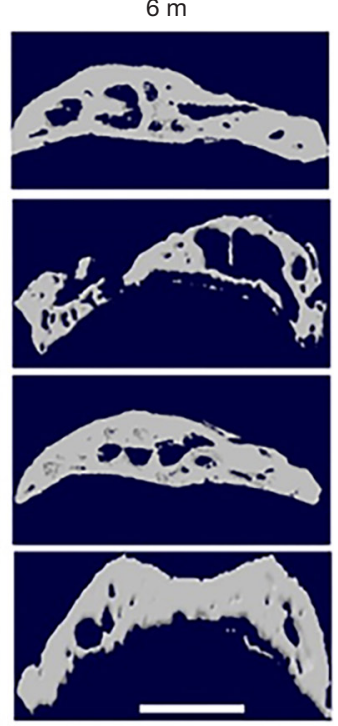

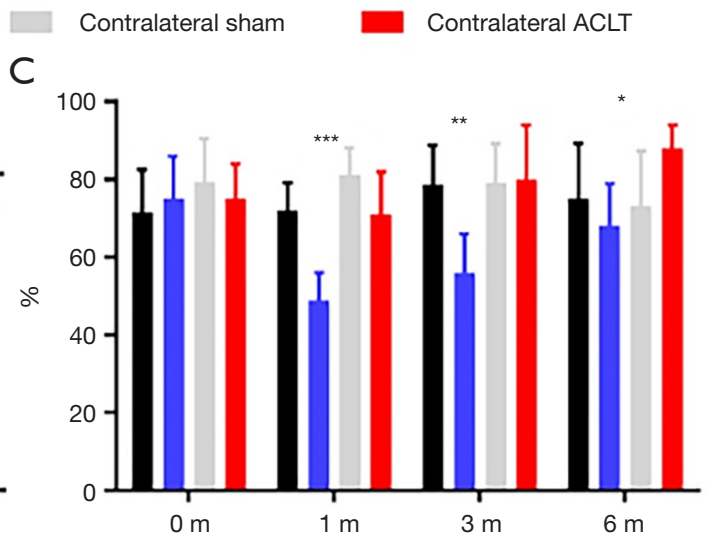

Figure 3 Differential subchondral bone architecture in ipsilateral and contralateral knees after ACLT. (A) Representative photos of CT 3D reconstruction of ipsilateral and contralateral tibial subchondral bone in different time points after ACLT; max contact max intensity (C) Statistical analysis of Tb Pf. (B) and BV/TV (C), n=8 per group. Statistical significance was determined by unpaired Student's $t$ test and all data were shown as a bar with means \pm standard deviations. Scale bar, $2 \mathrm{~mm}$; $, \mathrm{P}<0.05,{ }^{* *}, \mathrm{P}<0.01$, ***, $\mathrm{P}<0.001$ compared with the sham operated group at different time points. ACLT, anterior cruciate ligament transection.

however, osteoclastic activity remained nearly unchanged through OA progression and osteoblastic activity increased 3 months after ACLT. These data suggest both aberrant catabolic and anabolic changes could be harmful to articular cartilage in OA progression. Osteoblastic activity detected by Osterix signal was observed to peak at 1 month post surgery in the ipsilateral subchondral bone, whereas the Osterix signal gradually elevated throughout 6 months post surgery in the contralateral side (Figure 5). These findings suggest that instability and excessive mechanical loading caused OA through different mechanisms that involve osteoblastic and osteoclastic activity.

\section{Discussion}

OA was observed to develop in both knees and the current hypothesis for this phenomenon involves altered mechanical loading that accelerates disease progression $(24,25)$. In OA patients, it has been broadly observed that subchondral bone is abnormal, exhibiting osteoporosis and sclerosis within 

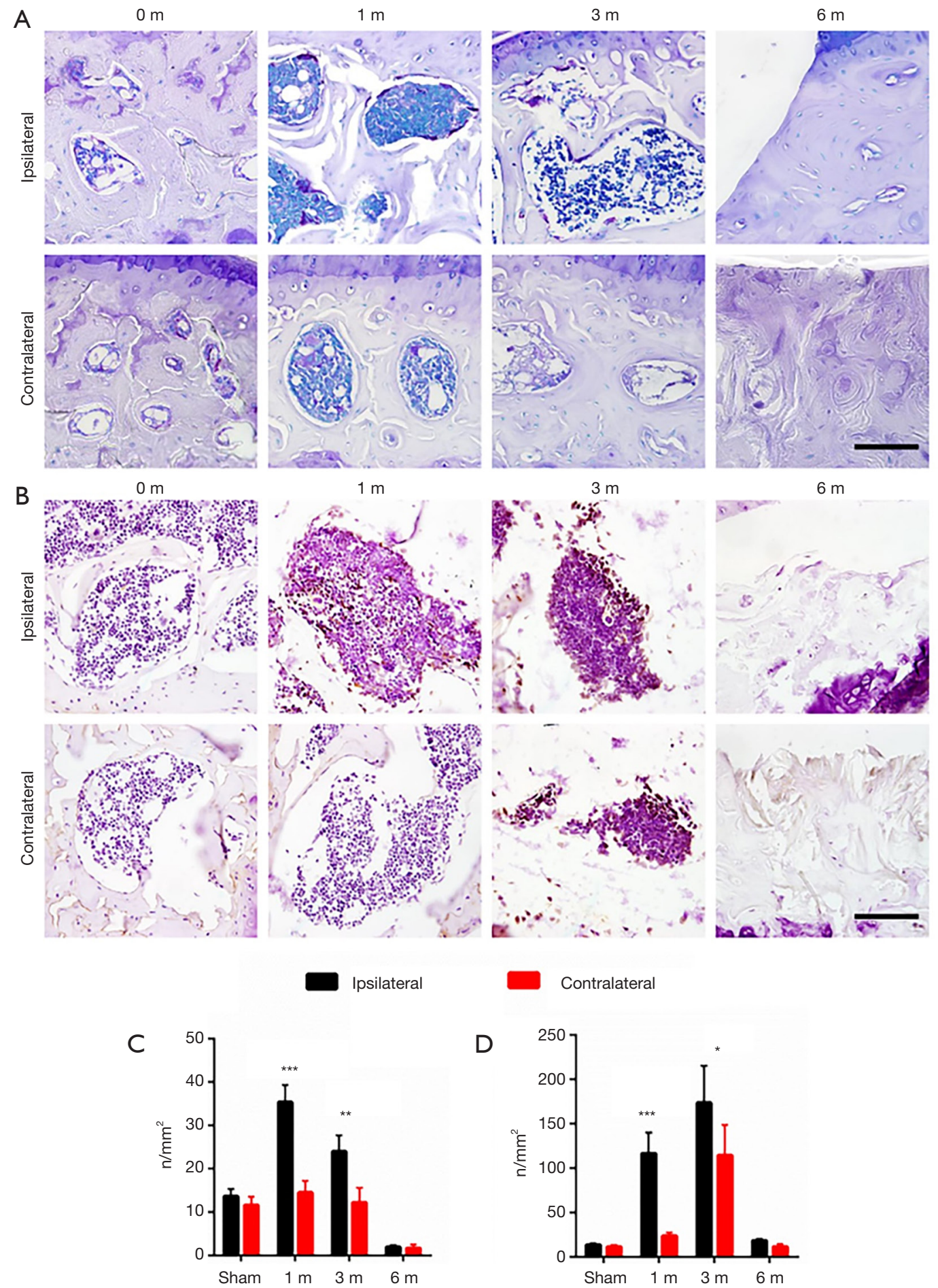

Figure 4 Differential osteoblastic and osteoclastic activities in ipsilateral and contralateral knees after ACLT. Representative photos of TRAP staining (A) and Osterix immunostaining (B) in ipsilateral and contralateral tibial subchondral bone in different time points after ACLT. statistical analysis of TRAP staining (C) and Osterix immunostaining (D), $n=8$ per group, statistical significance was determined by unpaired Student's $t$ test and all data were shown as a bar with means \pm standard deviations. Scale bar, $20 \mu \mathrm{m} ;{ }^{*}, \mathrm{P}<0.05,{ }^{* *}, \mathrm{P}<0.01,{ }^{* * *}$, $\mathrm{P}<0.001$ compared with the sham operated group at different time points. ACLT, anterior cruciate ligament transection. 


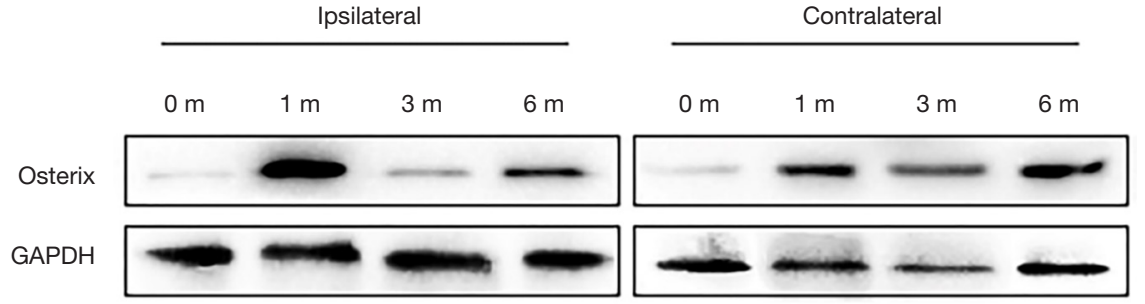

Figure 5 Western blot analysis of Osterix signal in ipsilateral and contralateral tibial subchondral bone in different time points after ACLT. ACLT, anterior cruciate ligament transection.

neighboring areas in the same joint (26). Our study aimed to explain how distinct mechanical changes differentially influenced OA progression through aberrant subchondral bone remodeling.

We found that ACLT caused an acute mechanical instability which resulted in an acute subchondral bone remodeling response and rapidly progressing OA. Simultaneously, gradual mechanical overloading of contralateral knees activated osteoblastic activity which caused subchondral bone sclerosis. These findings suggest that both osteoporosis and sclerosis of subchondral bone contribute to articular cartilage destruction in OA progression. The causal relationship and spatiotemporal relationship between subchondral bone remodeling and articular cartilage deterioration, however, remain largely unknown. Mechanical loading (4), aberrant remodeling and disturbed microcirculation $(27,28)$ could be responsible for this imbalance.

Mechanical loading can have multifunctional effects on subchondral bone structure and remodeling. Within a physiological range, mechanical loading is essential for bone metabolism and cartilage survival $(17,29)$; however, overloading of subchondral bone and cartilage can also lead to OA progression $(30,31)$. Consistent with previous studies, we observed increases in both osteoclastic and osteoblastic activity in response to acute mechanical instability induced by ACLT $(12,32)$. The net effect of these two antagonistic reactions was decreased subchondral bone volume at the early stage of OA, and a partial recovery at the late stage by uncoupled sclerosis. It was also noted that mechanical instability developed subchondral bone changes in the contralateral knee (17). As shown in our results, mechanical load shifting from the ipsilateral side to the contralateral side influences subchondral bone remodeling. Although acute mechanical instability was not exerted on contralateral side, the gait change caused by OA progression and pain development functionally shifted mechanical loading to the contralateral side. This gradual shift of mechanical loading changes the subchondral bone metabolism to osteoblastic bone formation.

Subchondral bone reacts differently to specific mechanical loading patterns. We find load-related increases in subchondral bone thickness, epiphyseal trabecular thickness, lateral joint compartment mass and subchondral bone thickening in the femur compared to the tibia after 5 weeks. This aligns with our previous finding from a non-invasive mouse model where only articular femoral lesions occur, that applied loads are transferred predominantly across the joint's lateral aspect (10). Our data are consistent with the conclusion that repetitive loads, applied directly across articular surfaces, can produce subchondral bone thickening in intact joints and that loadinduced subchondral bone thickening is further accentuated in regions underlying cartilage lesions. Our study reveals bone changes in the knee of the contralateral limb, with subchondral bone thickening evident at the early 2 -week time point (in animals loaded once or multiple times). We also show widespread increases in epiphyseal trabecular thickness and mass in non-loading contralateral joints after 5 weeks. Gait modifications affecting contralateral limbs were observed. In these limbs, bone changes occurred, suggesting that compensatory limb use underpins both the transient subchondral bone thickening observed at 2 weeks and the later accrual of underlying epiphyseal bone.

Relationships between articular cartilage lesions and subchondral bone remain unclear. It has been speculated that changes in subchondral bone stiffness predispose articular cartilage to fibrillation. It is also possible that articular cartilage damage itself accelerates subchondral bone remodeling and thickening by changing joint mechanics (18). Diverging opinions have mostly been informed by use of models where joint mechanics are intransigently altered by permanent surgical anatomical disruption. Our use of a model that allows for transient joint loading, in which load-induced cartilage lesions are created in only a single compartment, provides a different basis for determining temporospatial links. Our findings indicate that subchondral bone thickening can 
indeed be induced by applied loads beneath intact articular cartilage and that lesion-containing articular cartilage regions, which share applied loads, may act to accentuate this thickening. Moreover, since the stem cell recruitment to the subchondral bone is through TGF-b signaling upon mechanical loading, the mechanism of action of this rebalanced mechanical loading might be attributed to stem cell fate change in subchondral bone and articular cartilage $(12,33)$.

Increases in subchondral bone thickness and volume are known to occur in OA (4) and increased stiffness is thought to adversely affect articular cartilage biomechanics, leading to a loss of structural integrity. Epiphyseal bone changes are now known to accompany this subchondral bone thickening in mouse models of post-traumatic OA and destabilization of the medial meniscus. This latter model has also shown less subchondral bone thickening and decreased levels of articular cartilage damage in ADAMTS5-deficient mice, thus strengthening the association. Subchondral bone sclerosis has also been described in spontaneous OA models. Other studies have shown, however, that early timepoints following mechanical trauma can induce decreases in epiphyseal bone parameters (23). It is worth noting that joint injury induced in these earlier studies was more severe than in ours, with clear joint subluxation leading to severe OA.

Direct comparison between loaded and contralateral non-loaded knees in our analyses show corresponding decreases in subchondral bone thickness at early timepoints ( 2 weeks), which are reversed by 5 weeks. This emphasizes the need to use appropriate controls. Our study is somewhat limited by examining responses only at specific timepoints. Highly dynamic bone loading responses have been shown previously and caution should therefore be exercised when comparing each of the various parameters measured at any specific timepoint. In conclusion, our pioneering study provides us a new point of view for the systemic treatment of OA by indicating simultaneous prevention of ipsilateral and contralateral $\mathrm{OA}$ in the clinical practice.

\section{Acknowledgments}

Funding: This work was supported by national Key R\&D Program of China (2019YFA0111900), National Natural Science Foundation of China (No. 81874030), InnovationDriven Project of Central South university (No. 2020CX045), the Key program of Health Commission of Hunan Province (20201902), the Provincial Science Foundation of Hunan (No. 2018JJ2636), the Key Research and Development Program of Hunan Province (No. 2018SK2076) and CMA
Young and Middle-aged Doctors Outstanding Development Program--Osteoporosis Specialized Scientific Research Fund Project (G-X-2019-1107-12).

\section{Footnote}

Conflicts of Interest: YL serves as an unpaid section editor of Annals of Translational Medicine from Oct 2019 to Sep 2020. The other authors have no conflicts of interest to declare.

Ethical Statement: The authors are accountable for all aspects of the work in ensuring that questions related to the accuracy or integrity of any part of the work are appropriately investigated and resolved. All procedures complied with the Animals (Scientific Procedures) Act 1986 and received university ethical approval of Xiangya Hospital (2019030186).

Open Access Statement: This is an Open Access article distributed in accordance with the Creative Commons Attribution-NonCommercial-NoDerivs 4.0 International License (CC BY-NC-ND 4.0), which permits the noncommercial replication and distribution of the article with the strict proviso that no changes or edits are made and the original work is properly cited (including links to both the formal publication through the relevant DOI and the license). See: https://creativecommons.org/licenses/by-nc-nd/4.0/.

\section{References}

1. Hootman JM, Helmick CG. Projections of US prevalence of arthritis and associated activity limitations. Arthritis Rheum 2006;54:226-9.

2. Zhu J, Lei P, Hu Y. Intraarticular hyaluronate injection for knee osteoarthritis—reconsider the rationale. Ann Transl Med 2015;3:214.

3. Hunter DJ, Schofield D, Callander E. The individual and socioeconomic impact of osteoarthritis. Nat Rev Rheumatol 2014;10:437.

4. Griffin TM, Guilak F. The role of mechanical loading in the onset and progression of osteoarthritis. Exerc Sport Sci Rev 2005;33:195-200.

5. Read SJ, Dray A. Osteoarthritic pain: a review of current, theoretical and emerging therapeutics. Expert Opin Investig Drugs 2008;17:619-40.

6. Hochberg MC, Altman RD, April KT, et al. American College of Rheumatology 2012 recommendations for the use of nonpharmacologic and pharmacologic therapies in 
osteoarthritis of the hand, hip, and knee. Arthritis Care Res (Hoboken) 2012;64:465-74.

7. Saito T, Fukai A, Mabuchi A, et al. Transcriptional regulation of endochondral ossification by HIF-2 alpha during skeletal growth and osteoarthritis development. Nat Med 2010;16:678-86.

8. Wang Q, Rozelle AL, Lepus CM, et al. Identification of a central role for complement in osteoarthritis. Nat Med 2011;17:1674-9.

9. Coughlin TR, Kennedy OD. The role of subchondral bone damage in post-traumatic osteoarthritis. Ann N Y Acad Sci 2016;1383:58-66.

10. Anderson-MacKenzie JM, Quasnichka HL, Starr RL, et al. Fundamental subchondral bone changes in spontaneous knee osteoarthritis. Int J Biochem Cell Biol 2005;37:224-36.

11. Dare D, Rodeo S. Mechanisms of Post-traumatic Osteoarthritis After ACL Injury. Curr Rheumatol Rep 2014;16:448.

12. Zhen G, Wen CY, Jia XF, et al. Inhibition of TGF-beta signaling in mesenchymal stem cells of subchondral bone attenuates osteoarthritis. Nat Med 2013;19:704.

13. Arden N, Nevitt MC. Osteoarthritis: epidemiology. Best Pract Res Clin Rheumatol 2006;20:3-25.

14. Lim CT, Zhou EH, Quek ST. Mechanical models for living cells--a review. J Biomech 2006;39:195-216.

15. Findlay DM, Atkins GJ. Osteoblast-ChoWndrocyte Interactions in Osteoarthritis. Curr Osteoporos Rep 2014;12:127-34.

16. Felson DT, Chaisson CE, Hill CL, et al. The association of bone marrow lesions with pain in knee osteoarthritis. Ann Intern Med 2001;134:541-9.

17. Poulet B, de Souza R, Kent AV, et al. Intermittent applied mechanical loading induces subchondral bone thickening that may be intensified locally by contiguous articular cartilage lesions. Osteoarthritis Cartilage 2015;23:940-8.

18. Bian L, Angione SL, Ng KW, et al. Influence of decreasing nutrient path length on the development of engineered cartilage. Osteoarthritis Cartilage 2009;17:677-85.

19. Kapoor M, Martel-Pelletier J, Lajeunesse D, et al. Role of proinflammatory cytokines in the pathophysiology of osteoarthritis. Nat Rev Rheumatol 2011;7:33-42.

20. Li Y, Xiao W, Luo W, et al. Alterations of amino acid metabolism in osteoarthritis: its implications for nutrition and health. Amino Acids 2016;48:907-14.

21. Glasson SS, Chambers MG, Van den Berg WB, et al. The OARSI histopathology initiative - recommendations for histological assessments of osteoarthritis in the mouse. Osteoarthritis Cartilage 2010;18:S17-23.
22. Lakes EH, Allen KD. Gait analysis methods for rodent models of arthritic disorders: reviews and recommendations. Osteoarthritis Cartilage 2016;24:1837-49.

23. Zhu S, Zhu J, Zhen G, et al. Subchondral bone osteoclasts induce sensory innervation and osteoarthritis pain. J Clin Invest 2019;129:1076-93.

24. Wegener L, Kisner C, Nichols D. Static and dynamic balance responses in persons with bilateral knee osteoarthritis. J Orthop Sports Phys Ther 1997;25:13-8.

25. Shakoor N, Dua A, Thorp LE, et al. Asymmetric loading and bone mineral density at the asymptomatic knees of patients with unilateral hip osteoarthritis. Arthritis Rheum 2011;63:3853-8.

26. Hunter DJ, Hart D, Snieder H, et al. Evidence of altered bone turnover, vitamin $\mathrm{D}$ and calcium regulation with knee osteoarthritis in female twins. Rheumatology 2003;42:1311-6.

27. Xie H, Cui Z, Wang L, et al. PDGF-BB secreted by preosteoclasts induces angiogenesis during coupling with osteogenesis. Nat Med 2014;20:1270.

28. Aaron RK, Racine JR, Voisinet A, et al. Subchondral bone circulation in osteoarthritis of the human knee. Osteoarthritis Cartilage 2018;26:940-4.

29. Iijima H, Ito A, Nagai M, et al. Physiological exercise loading suppresses post-traumatic osteoarthritis progression via an increase in bone morphogenetic proteins expression in an experimental rat knee model. Osteoarthritis Cartilage 2017;25:964-75.

30. Neogi T, Nevitt M, Niu J, et al. Subchondral bone attrition may be a reflection of compartment-specific mechanical load: the MOST Study. Ann Rheum Dis 2010;69:841-4.

31. Iijima H, Aoyama T, Tajino J, et al. Subchondral plate porosity colocalizes with the point of mechanical load during ambulation in a rat knee model of post-traumatic osteoarthritis. Osteoarthritis Cartilage 2016;24:354-63.

32. $\mathrm{Xu} X$, Zheng LW, Bian Q, et al. Aberrant Activation of TGF- in Subchondral Bone at the Onset of Rheumatoid Arthritis Joint Destruction. J Bone Miner Res 2015;30:2033-43.

33. Freitag J, Bates D, Wickham J, et al. Adipose-derived mesenchymal stem cell therapy in the treatment of knee osteoarthritis: a randomized controlled trial. Regen Med 2019;14:213-30.

Cite this article as: Zhu J, Zhu Y, Xiao W, Hu Y, Li Y. Instability and excessive mechanical loading mediate subchondral bone changes to induce osteoarthritis. Ann Transl Med 2020;8(6):350. doi: 10.21037/atm.2020.02.103 